\title{
A dual-task analysis of detection accuracy for the case of high target-distractor similarity: Further evidence for independent processing
}

\author{
DAVID BURNS \\ McMaster University, Hamilton, Ontario L8S 4K1, Canada
}

\begin{abstract}
Previous studies of visual detection performance indicate that perceptual span has a negligible effect on detection accuracy; hence, previous results suggest that individual characters within a multielement array are perceived independently. In this study, additional experimental factors were examined in order to determine whether conditions exist in which perceptual span influences detection performance: targets and distractors were chosen to be maximally similar, and detection accuracy was studied in relation to display size, target redundancy, and homogeneity of distractor characters. To test for perceptual span effects, a three-state model was proposed which incorporates the assumption that individual characters are perceived independently. The model may be applied to (a) both yes-no and forced-choice tasks, and (b) the case when effects of display characteristics differ for response alternatives. Predictions based on the three-state model agree satisfactorily with observed proportions. Moreover, interactions between display characteristics and response alternatives accord well with model predictions, and accuracy differences between subjects are consistent with individual differences in model parameters. The results provide further evidence for the independence assumption and illustrate additional conditions for which perceptual span has a negligible effect on detection accuracy.
\end{abstract}

An early goal of experimental psychology was to develop a measure of perceptual span, defined as the upper bound on the number of characters a subject perceives within a limited time interval. Cattell (1883) proposed a "report"' measure of perceptual span: Using a tachistoscope, he presented an array of characters to subjects and instructed them to report as many as possible. At first glance, the report procedure appears to be a valid method for measuring perceptual span, since characters that are not perceived cannot be reported. Yet, it does not follow that characters which are perceived are necessarily reported, since delays exist between perception and response. For example, perceived characters may not be reported because of forgetting from short-term memory or limitations on vocalization rate (James, 1890; Sperling, 1960). To the extent that nonpercep-

Preparation of this article was supported by NIMH Grant MH 23878 to Rockefeller University, a Ford Foundation grant to Rockefeller University, and Grant A0229 from the National Research Council of Canada. Experimental work was supported by a Maryland Fellowship, the Biomedical Sciences Support Center, and the Computer Center of the University of Maryland. The author is grateful to N. S. Anderson and W. D. Larkin for assistance in the formulation of the problem, to John Holmgren and John Theios for assistance in model development, and to W. K. Estes, S. W. Link, D. L. Noreen, and A. B. Kristofferson for criticism of earlier drafts. Reprint requests should be sent to David Burns, BBDO, Inc., 383 Madison Avenue, New York, New York 10017. tual processes contribute to report performance, then, the procedure cannot be said to measure perceptual span per se; rather, report procedures measure combined performance for all processes which intervene between perception and report.

To reduce the contribution of forgetting to measures of perceptual span, Estes and Taylor (1964, 1966) proposed a measure based on a forced-choice detection task. In the detection task, subjects indicate which one of two possible targets occurs in a tachistoscopically presented array, and each array contains one target alternative, along with some number of distractors, (i.e., nontarget characters). Estes and Taylor derived their measure of perceptual span from a serial-processing model of detection which is based on two assumptions: (1) Individual characters are perceived serially, (i.e., one by one), at a constant rate, until no information remains in the sensory trace; and (2) if the target is detected, a correct response occurs with probability 1.0 and, if the target is not detected, a correct response occurs with a probability of .5. (Partial information, then, would not contribute to the subject's response.) From these assumptions, Estes and Taylor showed that: (1) detection accuracy, defined as the probability of a correct detection response, is directly related to the proportion of characters perceived, $p$; (2) p could be estimated by correcting observed values of detection accuracy for the effects of guessing; and (3) given an estimate 
of $p$, the number of characters perceived, $L$, may be estimated, since $\mathrm{L}=\mathrm{p} \cdot \mathrm{N}$, where $\mathrm{N}$ equals display size (i.e., the number of characters presented). Thus, the serial-processing model allows an estimation of $\mathrm{L}_{c}$, the perceptual span: The limiting value of $\mathrm{L}$ is $\mathrm{L}_{c}$ as $\mathbf{N}$ increases. Estes and Taylor observed that $\mathbf{L}$ tended to a limit when $\mathbf{N}$ was varied; that is, their results indicated that perceptual span places an upper limit on detection accuracy, in that no increase in number of characters perceived appeared to occur once display size exceeded perceptual span. Stated differently, their results indicated a display-size effect: detection accuracy decreased as display size increased. Estes and Taylor ascribed the effect to a decrease in proportion of characters perceived as $\mathbf{N}$ increases; if $\mathrm{p}$ were constant, no display-size effect would be expected.

Like Estes and Taylor, Rumelhart (1970) estimated $\mathrm{L}_{\mathrm{c}}$ after outlining an interpretation of the display-size effect in terms of perceptual-span limitations. However, Rumelhart assumed that characters are processed in parallel, and that, as $\mathrm{N}$ varies, the supply of attentional resources allocated to each display remains constant. In Rumelhart's multicomponent model, the display-size effect is assumed to result from a decrease in average perceptual clarity of individual characters as $\mathrm{N}$ increases, rather than from a decrease in proportion of characters identified, as assumed in the serial-processing model. Nevertheless, the two models agree inasmuch as identification. probability, defined as the average probability of identifying any one character, is assumed to decrease as $\mathbf{N}$ increases. Because both models ascribe decreases in identification probability solely to limitations of perceptual span, they have been classified as limitedcapacity models (Gardner, 1973).

The contrary proposition to the limited-capacity hypothesis is the independence assumption: Identification probabilities remain constant as $\mathrm{N}$ varies. Eriksen and Spencer (1969) recognized that, although a display-size effect would not be expected on the basis of the independence assumption, the effect would be expected if the independence assumption were combined with a confusion-error hypothesis, i.e., the hypothesis that the probability of identification errors is nonzero. A decrease in detection accuracy would be expected to result from confusion errors, since, as $\mathrm{N}$ increased, the probability would increase that at least one distractor would be misidentified as a target. The occurrence of confusion errors would not imply independent processing, however; confusion errors could occur whether the independence assumption were true or false. Therefore, there are three distinct hypotheses to consider: First, the display-size effect may be due to span limitations in the absence of confusion errors, as stated in the limited-capacity hypotheses. Second, the effect may result from confusion errors in the absence of span limitations, as proposed by Eriksen and Spencer. Third, both span limitations and confusion errors may contribute to the effect.

As the matter stands at this point in the account of perceptual-span measures, the validity of Estes and Taylor's and Rumelhart's estimates of $L_{c}$ is related to the origin of the display-size effect: To the extent that the effect is due to span limitations, estimates of $L_{c}$ are expected to be valid. However, the existence of confusion errors would necessitate at least a correction of Estes and Taylor's procedure for estimating $L_{c}$, if not a rejection of the limitedcapacity hypothesis in favor of the independence assumption.

To test for the existence of confusion errors, then, Eriksen and Spencer (1969) investigated the effects of confusability between distractors and a single target character. For this purpose, they developed the yes-no detection task: A single character was designated as the target and, on each trial, the stimulus array either did or did not contain a target. Subjects indicated whether or not the target was perceived. If confusion errors contribute to the display-size effect (in this case, defined as a decrease in the proportion of correct responses averaged over "yes" and "no" responses), then, as $N$ increases, the probability increases that at least one distractor will be mistaken as the target (provided that targets and distractors are confusable and response criteria remain constant). Thus, the probability of a "yes" response is expected to increase as display size increases. Because such a response pattern would decrease the probability of missing a target, a target-array effect is expected as well: The probability of a correct "yes" response is expected to increase as display size increases. A target-array effect is not expected on the basis of the limited-capacity hypothesis alone, however, since, as $\mathbf{N}$ increases, detection accuracy is expected to decrease for both "yes" and "no" responses (provided response criteria remain constant).

Eriksen and Spencer observed both a display-size effect and a target-array effect. Accordingly, they concluded that, in their experiment, confusion errors necessarily contributed to detection performance, and that the limited-capacity hypothesis is insufficient to account for their results. However, their results need not imply that confusion errors are sufficient to account for the display-size effect; the possibility exists that any decrease in detection probability resulting from span limitations was obscured by an increase in detection probability due to confusion errors. For this reason, subsequent research has focused on the sufficiency of the independentchannels-confusion hypothesis: Does the display-size 
effect result from a combination of span limitations and confusion errors, or are confusion errors the only factor?

Three experimental results have been proposed as evidence for the sufficiency of the independentchannels-confusion hypothesis. First, Eriksen and Spencer investigated the independence hypothesis by varying interstimulus interval (ISI); they compared the usual mode of presentation, in which individual characters appeared to occur simultaneously, to a condition in which characters were presented successively. Presentation time for each character remained constant for the two conditions, but, in the successive condition, a longer ISI occurred between presentations of individual characters. Eriksen and Spencer maintained that if perceptual span limits performance in the simultaneous condition, then detection accuracy should increase as ISI increases, since the number of characters identified is expected to increase as ISI increases. However, if the independence assumption is true, no effect of ISI was expected, since the number of characters perceived is not assumed to depend on the rate of identification of characters.

Eriksen and Spencer varied ISI from $5 \mathrm{msec}$ to $3 \mathrm{sec}$ and observed no changes in detection accuracy. Accordingly, Eriksen and Spencer and others (Shiffrin \& Gardner, 1972) concluded that span limitations do not contribute to detection performance. Strictly speaking, however, the absence of an effect for ISI need not imply that perceptual span does not affect detection accuracy. Rather, the absence of an effect for ISI may indicate only that span limitations remained constant as ISI varied, e.g., if subjects allocated a constant amount of processing resources to the task, regardless of ISI. So, while span limitations may impose no limit on detection performance in Eriksen and Spencer's experiment, the possibility exists that span limitations have a constant effect on detection accuracy as ISI varies. Therefore, the absence of an effect for ISI need not imply that confusion errors are solely responsible for the displaysize effect.

A second source of evidence regarding the sufficiency of the independent-channels-confusion hypothesis results from varying the similarity between target and distractor characters (Estes, 1972; Gardner, 1973). If both confusion errors and span limitations contribute to the display-size effect, and if perceptual span remains constant as similarity varies, then a display-size effect is expected in both high- and lowsimilarity conditions: a display-size effect is expected in the high-similarity condition, since both confusion errors and span limitations affect detection accuracy. In addition, a display-size effect is expected in the low-similarity condition because of span limitations. In contrast, if confusion errors are solely responsible for the display-size effect in the high-similarity condi- tion, then the display-size effect is expected to be negligible in the low-similarity condition, since the effects of confusion errors are assumed to be negligible in the low-similarity condition. Both Estes' and Gardner's results indicate that a significant display-size effect occurs when targets and distractors are similar, while the effect is insignificant when targets and distractors are sufficiently dissimilar.

If perceptual span remained constant in these experiments, then the conclusion follows that the independent-channels-confusion hypothesis is sufficient to account for the display-size effect. Yet, the hypothesis that perceptual span remains constant is subject to question if one considers the effects of target-distractor similarity on resource allocation. Norman and Bobrow (1975) hypothesized that, as $\mathbf{N}$ increases, attentional or perceptual resources continue to be allocated until all available resources, $\mathbf{R}_{c}$, are expended. In addition, Norman and Bobrow assumed that the slope of the function relating resource allocation to $\mathbf{N}$ increases as target-distractor similarity increases (Figure 1). On the basis of Norman and Bobrow's analysis, display sizes exist, $\mathrm{L}_{\mathrm{c}_{1}}<\mathrm{N}<\mathrm{L}_{\mathrm{c}_{2}}$, such that span limitations occur when similarity is high, but not when similarity is low. Stated differently, $\mathrm{L}_{\mathrm{c}_{1}}$, the span for the high-similarity condition may be less than $\mathrm{L}_{\mathrm{c}_{2}}$, the span for the low-similarity condition. The possibility exists, then, that perceptual span varies as a function of target-distractor similarity and, for this reason, the observation that the displaysize effect occurs only for similar target-distractor pairs need not imply that confusion errors are sufficient to account for the display-size effect.

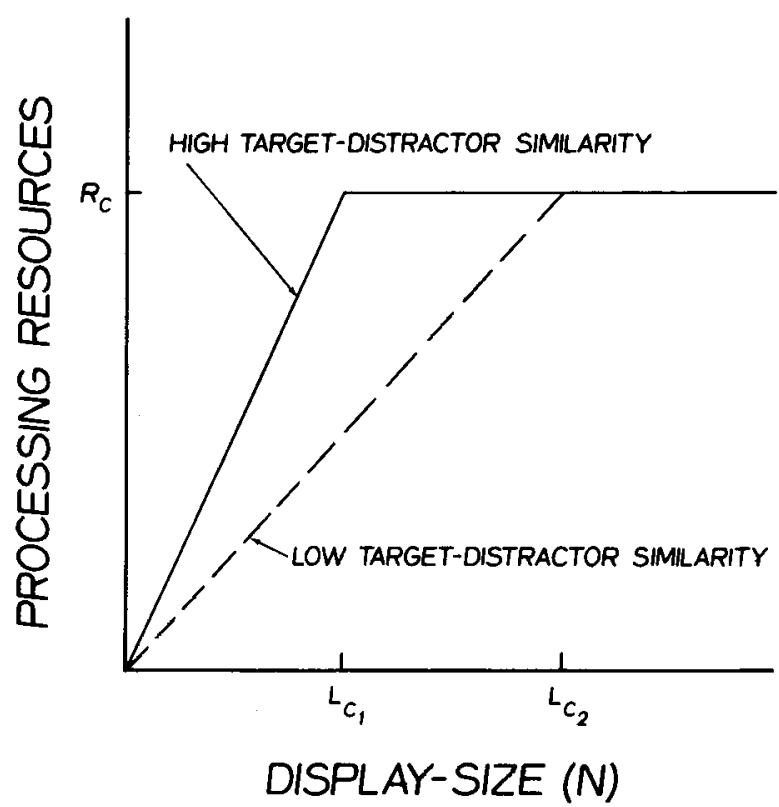

Figure 1. Illustration of a possible relation between resourceallocation and target-distractor similarity. 
The third source of evidence regarding the sufficiency of the independent-channels-confusion hypothesis is based on goodness-of-fit tests of a specific model of the detection process. According to the goodness-of-fit strategy, a detection model is developed which specifies the expected effects of confusion errors on detection accuracy, provided that the independence assumption is true. Observed values of detection accuracy are compared to predictions based on the independence assumption, in combination with the specific detection model. If satisfactory agreement is obtained, as measured by goodness-offit statistics, support is inferred for both the independence assumption and the specific detection model.

The results for all three studies employing the goodness-of-fit strategy (Estes, 1976; Kinchla, 1974; Shiffrin \& Geisler, 1973) indicate satisfactory agreement between observed and predicted values of detection accuracy. Hence, the independence hypothesis in combination with some specific model of the detection process appears sufficient to describe existing display-size effects. Still, since previous tests of the independence hypothesis employed (1) relatively few characters (Gardner, 1973), or (2) distractor characters from a heterogeneous set (Eriksen \& Spencer, 1969; Estes, 1976), further research is needed to identify any experimental conditions for which span limitations might exist.

Further research is also needed to generalize existing detection models, since they apply either to forcedchoice (Estes, 1976; Shiffrin \& Geisler, 1973) or yes-no tasks (Kinchla, 1974), but not to both. Because the independence assumption may be true for one task but not the other (Kinchla, 1974), it is of interest to observe detection performance in both tasks under similar conditions and apply the same detection model to the results for both tasks. Moreover, existing detection models apply only to results averaged over stimuli (or responses). But, because the average results may be unrepresentative of the effects observed for individual stimulus/response pairs, it is of interest to develop models which apply to the data for each stimulus/response pair individually.

In the current study, the goodness-of-fit strategy was employed in order to test the generality of existing detection results; the model proposed here was designed to apply to yes-no as well as forced-choice tasks, and to the case in which distinct effects are observed for the two stimuli (target types). In addition, display size was varied over the largest possible range while maintaining constant acuity and minimal lateral interference, and distractor characters were chosen to be maximally similar to targets, as determined by the confusion probabilities estimated in visual-identification experiments (Fisher, Monty, \& Glucksberg, 1969; Townsend, 1971).

\section{A Three-State Model}

In the model proposed here, two successive stages of the detection process are outlined: encoding and response selection. As a result of encoding, each character is classified into one of three mutually exclusive and exhaustive encoding states, which are defined with respect to their effects on response tendency. For example, the classification of a character into state $X_{1}$ is said to increase the observer's tendency to respond, $R_{\mathbf{l}}$.

The classification of any one character, though, is insufficient for response selection; response selection depends on the combined effect of classifying all characters. For this reason, a rule is needed for selecting a response, provided that each character has been classified. In the response-selection stage of the proposed model, all possible combinations of encoding states are enumerated and classified into decision states. Then a response rule is introduced which specifies the probability of each response conditional on the particular decision state.

\section{Encoding Assumption}

The three-state model is similar to several existing detection models (Atkinson \& Kinchla, 1965; Gardner, 1973; Holmgren, 1968) inasmuch as the outcomes of perceptual processing are partitioned into three mutually exclusive and exhaustive encoding states, $X_{j}, j=0,1,2$. If the result of processing an individual character influences the observer's response tendency toward $R_{m}(m=1,2)$, the character is said to be classified into state $X_{m}$. If the result of processing an individual character influences the observer's response tendency toward neither $\mathbf{R}_{1}$ nor $\mathbf{R}_{\mathbf{2}}$, the perceptual representation for that character is said to be classified into state $X_{0}$.

The set of $\mathbf{n}$ characters included in an experiment is designated by $\left\{s_{i}\right\}, i=1,2 \ldots, n$ (thus, if $n=3$, only three characters would ever be included in an array). The first numerals in the series $(1,2, \ldots, n)$ designate targets, and the remainder designate distractors. Thus, for the yes-no task, $s_{1}$ denotes the target, whereas for the forced-choice task both $s_{1}$ and $s_{2}$ denote targets. The classification probability, $\mathrm{p}_{\mathrm{ij}}$, is defined as the probability that the perceptual representation for $s_{i}$ is classified into state $X_{j}, j=0$, $1,2$.

The classification assumption proposed here differs from existing models in that the classification probabilities are estimable for each individual character. As a result of this assumption, the model makes predictions for both responses and for each distinct combination of stimuli.

\section{Independence Assumption}

Within an experiment, the endstate probabilities 
are assumed to depend only on the identity of $s_{i}$ and the endstate $X_{j}$. However, across experiments, the $p_{i j}$ may depend on the character set $s_{i}$. The $p_{i j}$ are assumed to be independent of display size, the number of occurrences of $s_{i}$ for all $i$, and the position of $s_{i}$ in the display.

\section{Decision Assumptions}

Because the assignment of a response to each decision state is task-specific, i.e., response rules differ for yes-no and forced-choice, the exact definition of decision states will be deferred to the sections specific to each task. However, for both tasks, it is the case that three mutually exclusive and exhaustive decision states, $D_{k}, k=0,1,2$, are postulated. State $D_{k}, k=1,2$, is said to be determinate, in that the effect of encoding is to influence the observer's tendency toward $R_{k}$ and not toward the alternative response $R_{j}(j=3-k)$. State $D_{0}$ is said to be indeterminate, in that the effect of encoding has no influence on the observer's response tendency.

\section{Response Rule}

If event $D_{k}$ occurs $(k=1,2)$, response $R_{k}$ occurs with probability 1.0 , whereas if event $D_{0}$ occurs, response $R_{1}$ is made with probability $\alpha$, where $\alpha$ represents response bias. This response rule leads to the following expressions for response probability:

$$
P\left(R_{1}\right)=P\left(D_{1}\right)+\alpha P\left(D_{0}\right)
$$

and

$$
\mathrm{P}\left(\mathbf{R}_{2}\right)=\mathrm{P}\left(\mathrm{D}_{2}\right)+(1-\alpha) \mathrm{P}\left(\mathrm{D}_{0}\right) .
$$

\section{The Yes-No Task}

For the yes-no task, the detection of a target (i.e., classification of any one character into state $X_{1}$ ) is sufficient, in principle, to indicate target presence. In contrast, for the conditions considered here, the detection of any one nontarget is uninformative with respect to response selection (with the exception of $\mathbf{N}=1$ ), since every display containing multiple characters contains nontargets, whether or not the target is present. For this reason, a determinate $D_{1}$ is assumed to occur if and only if at least one character is classified into state $\mathbf{X}_{1}$, regardless of the classifications for other characters.

Although several alternative definitions of $D_{2}$ and $D_{0}$ are possible, ${ }^{1}$ one reasonable assumption is that ambiguity regarding the classification of any one character is likely to lead to uncertainty in response selection. This assumption leads to the following definition of the indeterminate state: $D_{0}$ is said to occur whenever at least one character is ambiguous (i.e., classified into state $\mathrm{X}_{0}$ ). Given the above defini- tions for $D_{1}$ and $D_{0}$, state $D_{2}$ must occur whenever all characters are classified into state $\mathrm{X}_{2}$.

These definitions lead to the following expressions for decision-state probabilities, for the case when $\mathrm{n}=2$ :

$$
\begin{gathered}
P\left(D_{1} / S_{i}\right)=\left(1-p_{i 1}\right)^{K}\left(1-p_{21}\right)^{N-K} \\
P\left(D_{2} / S_{i}\right)=P_{i 2} K_{p_{22}} N-K
\end{gathered}
$$

and

$$
P\left(D_{0}\right)=1-P\left(D_{1}\right)-P\left(D_{2}\right)
$$

where $\mathrm{N}=$ display size and $\mathrm{K}=$ the number of occurrences of the target.

\section{The Forced-Choice Task}

In contrast to yes-no, the detection of any one character as the target in the forced-choice task (i.e., classification of a character into states $X_{1}$ or $X_{2}$ ) is never sufficient to preclude the alternative response. In fact, the existence of misclassifications implies that multiple characters may register as targets, and thus targets may be "detected" (e.g., one character is classified into state $X_{1}$, and a second character is classified into state $X_{2}$ ), even though a single target character is presented. For this reason, the determinate decision states must be defined conjunctively for the forced-choice task. That is, state $D_{1}$ is said to occur whenever at least one character is classified into state $X_{1}$ (event $d_{1}$ ) and no characters are classified into state $X_{2}$ (event $\sim d_{2}$ ). Similarly, the decision process is said to be in determinate state $D_{2}$ whenever at least one character is classified into state $X_{2}$ (event $d_{2}$ ) and no characters are classified into state $X_{1}$ (event $\sim d_{1}$ ). The decision process is said to be indeterminate (state $D_{0}$ ) if (a) encoding is ambiguous, i.e., no characters classified as $X_{1}$, and no characters classified as $X_{2}$ (event $\left[\sim d_{1}\right.$ and $\left.\sim \mathrm{d}_{2}\right]$ ), or (b) stimulus encoding results in conflicting information, i.e., at least one character is classified as $\mathrm{X}_{1}$ and at least one character is classified as $\mathrm{X}_{2}$ (event $\left[d_{1}\right.$ and $\left.d_{2}\right]$ ).

$$
\begin{aligned}
P\left(D_{j} / S_{i}\right)= & P\left(d_{j} \text { and } \sim d_{k} / S_{i}\right) \\
= & P\left(\sim d_{k} / S_{i}\right)-P\left(\sim d_{j} \text { and } \sim d_{k} / S_{i}\right) \\
= & \left(1-p_{i k}\right)^{K}\left(1-p_{3 k}\right)^{N-K} \\
& -\left(1-p_{i j}-p_{i k}\right)^{K}\left(1-p_{3 j}-p_{3 k}\right)^{N-K},
\end{aligned}
$$

where $\mathrm{i}, \mathrm{j}=1,2$, and $\mathrm{k}=3-\mathrm{j}, \mathrm{N}=$ display size, and $K=$ the number of occurrences of the target. Similarly, 


$$
\begin{aligned}
& P\left(D_{0} / S_{i}\right) \\
& =P\left(d_{1} \text { and } d_{2} / S_{i}\right)+P\left(\sim d_{1} \text { and } \sim d_{2} / S_{i}\right) \\
& =P\left(d_{1} / S_{i}\right)-P\left(\sim d_{2} / S_{i}\right)+2 P\left(\sim d_{1} \text { and } \sim d_{2} / S_{i}\right) \\
& =1-\left(1-p_{i 1}\right)^{K}\left(1-p_{31}\right)^{N-K}-\left(1-p_{i 2}\right)^{K}\left(1-p_{32}\right)^{N-K} \\
& \quad+2\left(1-p_{i 1}-p_{i 2}\right)^{K}\left(1-p_{31}-p_{32}\right)^{N-K}
\end{aligned}
$$

In the context of the three-state model, the question of whether or not perceptual span is limited may be rephrased in terms of whether or not the classification probabilities $\left(\mathrm{p}_{\mathrm{ij}}\right)$ remain constant as display size varies. If the $\mathrm{p}_{\mathrm{ij}}$ remain constant, the probabilities of identifying individual characters may be expected to remain constant, and perceptual span may be said to place no limits on detection performance. In contrast, if the probabilities of identifying individual characters decrease as $\mathrm{N}$ increases, then perceptual span may be said to limit detection performance. To test whether or not perceptual span is limited, then, observed response proportions may be compared to predicted proportions based on Equations 1-7 since, to derive Equations 1-7, it was assumed that the endstate probabilities remain constant as display characteristics (e.g., $\mathbf{N}$ and $\mathrm{K}$ ) vary. To the extent that the $\mathrm{p}_{\mathrm{ij}}$ remain constant and the assumptions of the threestate model accurately reflect the detection process, the predicted proportions based on Equations 1-7 are expected to approximate the observed proportions.

\section{METHOD}

\section{Stimuli and Apparatus}

Character arrays were illuminated by means of a scientific prototype tachistoscope (Model GB), fitted for binocular viewing. A response box, consisting of two telegraph keys mounted on a wooden frame, was placed beneath the viewer of the tachistoscope. Displays were constructed from photographs of uppercase letters positioned on the circumference of an imaginary circle which subtended $2^{\circ}$ visual angle. Letter arrays were centered within the visual field. Each letter appeared in the upright position, and subtended $.2^{\circ}$ in height. The minimum (center-to-center) displacement equaled $.75^{\circ}$, a distance sufficient to preclude lateral masking (Bouma, 1970; Collins \& Eriksen, 1967).

\section{Subjects}

Four graduate students enrolled at the University of Maryland served as subjects, and were paid $\$ 3 / \mathrm{h}$ for their participation. Two of the subjects participated in the yes-no task, and two participated in the forced-choice task.

\section{Procedure}

After adapting to the reduced lighting in the experimental room, the subjects were instructed to focus on a fixation point (field luminance $=15.4 \mathrm{~cd} / \mathrm{m}^{2}$ ) displayed in one field of the tachistoscope. The subjects initiated each trial by activating a knee switch; then, after a $1 / 2-\mathrm{sec}$ interval, the stimulus display (luminance = $86.1 \mathrm{~cd} / \mathrm{m}^{2}$ ) was illuminated. The fixation and stimulus fields differed in intensity in order to direct subjects' attention to the onset of the stimulus. The visual field was darkened for $1 \mathrm{sec}$ while the subject responded.

Exposure durations were adjusted such that the probability of a correct response, averaged over the first six sessions, equaled approximately .75 for each subject. The resulting exposure durations for the four subjects were $6,7,8$, and $11 \mathrm{msec}$.

A rest interval occurred halfway through the session, while slide trays were changed. The subjects participated in a total of 20 sessions, but the data from the first 6 sessions of the experiment and the first 10 trials of each session were not included in the data analyses.

The subjects were instructed to respond as quickly as possible while maintaining accuracy; they received no feedback concerning the correctness or speed of their responses. Both response accuracy and response time were recorded, but only the accuracy results are reported here. (Response times are reported in the appendix.)

\section{The Yes-No Task}

The letter " $C$ " was designated as target. One response key (labeled "Yes") was used to indicate detection of the target, and the other (labeled "No") was used to indicate that the target was not detected. Half of the displays (symbolized by $S_{1}$ ) contained one occurrence of the target, and half ( $S_{2}$ displays) contained no targets. Displays contained $1,2,4,6$, or 8 letters, arranged randomly in each of eight positions.

Two levels of homogeneity were studied: In the homogeneous condition, the letter " $G$ " served as the only distractor, whereas, in the heterogeneous condition, both " $O$ " and " $G$ "' served as distractors. Whenever the number of distractors was even for the heterogeneous condition, the numbers of $O s$ and Gs were equal, but whenever the number of distractors was odd, the number of Os exceeded the number of Gs by one.

If a complete factorial design had been employed, a total of 20 conditions would result by combining the five levels of display size, two display types $\left(S_{1}\right.$ or $\left.S_{2}\right)$, and two levels of homogeneity. However, target displays for $K=1$ contain no distractors, and, as a result, the two homogeneity conditions were indistinguishable when $\mathbf{K}=1$. Rather than doubling the number of observations for $S_{1}$ displays containing one letter, though, the number of observations for this condition was held constant, and the number of homogeneous $S_{1}$ displays containing more than one letter was increased, thus equating the number of target and nontarget displays.

Within each session, eight observations per cell were recorded for all display types except heterogeneous $S_{1}$ displays $(K \neq 1)$, which occurred 10 times per session. Hence, a total of 160 trials were recorded per session. Within each session, all conditions were randomly ordered from trial to trial.

\section{The Forced-Choice Task}

The letters " $C$ " and " $G$ " were designated as targets. One response key (labeled " $C$ ") was closed to indicate the detection of a " $C$ " $\left(R_{1}\right)$, and the other key (labeled " $G$ ") was closed to indicate the detection of a " $G$ " $\left(R_{2}\right)$. Half of the stimulus arrays contained at least one " $C$ " ( $\left(S_{1}\right.$ displays), half contained at least one " $G$ " ( $S_{2}$ displays), and no display contained both " $C$ ", and "G." Arrays contained 1, 2, 4, 6, or 8 letters. In addition, target redundancy (i.e., the number of occurrences of the target) was varied; displays contained 1, 2, or 3 targets.

The letter "O" served as the only distractor for the homogeneous condition, whereas both " $O$ " and " $Q$ " served as distractors for the heterogeneous condition. Whenever the number of distractors was even for the heterogeneous condition, the numbers of Os and Qs were equal, but whenever the number of distractors was odd, the number of Qs exceeded the number of Os by one.

If a complete factorial design had been employed, a total of 24 display types would result by combining the 12 possible display-size and target-redundancy conditions and the two levels of homogeneity. However, not all of the resulting 24 display types are 
distinct. Hence, only nine combinations of $\mathrm{N}$ and $\mathrm{K}$ were included in the heterogeneous condition. Thus, a total of 21 distinct display conditions were included for the forced-choice task, and if the differences between target type $\left(S_{1}\right.$ vs. $\left.S_{2}\right)$ are considered, a total of 42 conditions results. Four observations per condition were recorded within each session, for a total of 168 trials recorded per session.

All observations were included in the data analysis, even though a few responses occurred after the $1-s e c$ response interval. Within each session, all conditions varied randomly from trial to trial. The subjects were not informed of the variables being studied, but they were told that each display contained either a " $\mathrm{C}$ " or a "G," but not both.

\section{RESULTS}

The proportion of correct responses for each task are presented in Tables 1 and 2 . For the yes-no task, the effect of display size was significant ${ }^{2}$ when detection accuracy was averaged over response alternatives $(\mathrm{Z}=5.6, \mathrm{p}<.001, \mathrm{MSE}=.013)$, and a significant interaction was observed between display size and response alternative $(Z=7.1, p<.001, \mathrm{MSE}=$ $.013)$. Further analysis indicated that the effect of display size was significant for "no" responses

Table 1

Proportion of Correct Responses for the Yes-No Task, and Predictions Based on the Three-State Model

\begin{tabular}{|c|c|c|c|c|c|c|c|c|c|c|}
\hline & \multicolumn{10}{|c|}{ Display Size } \\
\hline & \multicolumn{5}{|c|}{ Target Present } & \multicolumn{5}{|c|}{ Target Absent } \\
\hline & 1 & 2 & 4 & 6 & 8 & 1 & 2 & 4 & 6 & 8 \\
\hline $\begin{array}{l}\text { Subject } 1 \\
\text { Observed } \\
\text { Predicted }\end{array}$ & $\begin{array}{l}.83 \\
.82\end{array}$ & $\begin{array}{l}.84 \\
.82\end{array}$ & $\begin{array}{l}.81 \\
.82\end{array}$ & $\begin{array}{l}.85 \\
.82\end{array}$ & $\begin{array}{l}.75 \\
.82\end{array}$ & $\begin{array}{l}.97 \\
.97\end{array}$ & $\begin{array}{l}.90 \\
.93\end{array}$ & $\begin{array}{l}.90 \\
.88\end{array}$ & $\begin{array}{l}.87 \\
.82\end{array}$ & $\begin{array}{l}.73 \\
.77\end{array}$ \\
\hline $\begin{array}{l}\text { Subject } 2 \\
\text { Observed } \\
\text { Predicted }\end{array}$ & $\begin{array}{l}.71 \\
.67\end{array}$ & $\begin{array}{l}.63 \\
.70\end{array}$ & $\begin{array}{l}.74 \\
.74\end{array}$ & $\begin{array}{l}.78 \\
.78\end{array}$ & $\begin{array}{l}.84 \\
.82\end{array}$ & $\begin{array}{l}.87 \\
.86\end{array}$ & $\begin{array}{l}.82 \\
.74\end{array}$ & $\begin{array}{l}.55 \\
.55\end{array}$ & $\begin{array}{l}.41 \\
.42\end{array}$ & $\begin{array}{l}.31 \\
.32\end{array}$ \\
\hline $\begin{array}{l}\text { Number of Observations } \\
\text { per Subject }\end{array}$ & 112 & 252 & 252 & 252 & 252 & 224 & 224 & 224 & 224 & 224 \\
\hline
\end{tabular}

Table 2

Proportion of Correct Responses for the Forced-Choice Task*, and Predictions Based on the Three-State Model

\begin{tabular}{|c|c|c|c|c|c|c|c|c|c|c|c|}
\hline \multirow{3}{*}{\multicolumn{2}{|c|}{$\begin{array}{c}\text { Target } \\
\text { Redundancy }\end{array}$}} & \multicolumn{10}{|c|}{ Display Size } \\
\hline & & \multicolumn{5}{|c|}{ Target = "C" } & \multicolumn{5}{|c|}{ Target = "G" } \\
\hline & & 1 & 2 & 4 & 6 & 8 & 1 & 2 & 4 & 6 & 8 \\
\hline & & \multicolumn{10}{|c|}{ Homogeneous } \\
\hline 1 & $\begin{array}{l}\text { Subject } 3 \\
\text { Subject } 4 \\
\text { Average } \\
\text { Prediction }\end{array}$ & $\begin{array}{l}.77 \\
.87 \\
.82 \\
.81\end{array}$ & $\begin{array}{l}.75 \\
.68 \\
.71 \\
.78\end{array}$ & $\begin{array}{l}.65 \\
.65 \\
.65 \\
.72\end{array}$ & $\begin{array}{l}.62 \\
.61 \\
.61 \\
.68\end{array}$ & $\begin{array}{l}.66 \\
.64 \\
.65 \\
.65\end{array}$ & $\begin{array}{l}.64 \\
.57 \\
.61 \\
.72\end{array}$ & $\begin{array}{l}.70 \\
.75 \\
.72 \\
.71\end{array}$ & $\begin{array}{l}.84 \\
.65 \\
.75 \\
.69\end{array}$ & $\begin{array}{l}.70 \\
.54 \\
.62 \\
.67\end{array}$ & $\begin{array}{l}.71 \\
.62 \\
.67 \\
.65\end{array}$ \\
\hline 2 & $\begin{array}{l}\text { Subject } 3 \\
\text { Subject } 4 \\
\text { Average } \\
\text { Prediction }\end{array}$ & & $\begin{array}{l}.95 \\
.96 \\
.96 \\
.91\end{array}$ & $\begin{array}{l}.79 \\
.86 \\
.82 \\
.85\end{array}$ & $\begin{array}{l}.93 \\
.86 \\
.89 \\
.79\end{array}$ & $\begin{array}{l}.77 \\
.76 \\
.77 \\
.75\end{array}$ & & $\begin{array}{l}.85 \\
.73 \\
.79 \\
.79\end{array}$ & $\begin{array}{l}.79 \\
.80 \\
.79 \\
.75\end{array}$ & $\begin{array}{l}.69 \\
.66 \\
.68 \\
.72\end{array}$ & $\begin{array}{l}.62 \\
.70 \\
.66 \\
.68\end{array}$ \\
\hline \multirow[t]{2}{*}{3} & $\begin{array}{l}\text { Subject } 3 \\
\text { Subject } 4 \\
\text { Average } \\
\text { Prediction }\end{array}$ & & & $\begin{array}{l}.86 \\
.89 \\
.87 \\
.92\end{array}$ & $\begin{array}{l}.85 \\
.70 \\
.77 \\
.86\end{array}$ & $\begin{array}{l}.91 \\
.87 \\
.89 \\
.81\end{array}$ & & & $\begin{array}{l}.79 \\
.80 \\
.79 \\
.76\end{array}$ & $\begin{array}{l}.74 \\
.67 \\
.71 \\
.72\end{array}$ & $\begin{array}{l}.62 \\
.64 \\
.63 \\
.68\end{array}$ \\
\hline & & \multicolumn{10}{|c|}{ Heterogeneous } \\
\hline 1 & $\begin{array}{l}\text { Subject } 3 \\
\text { Subject } 4 \\
\text { Average } \\
\text { Prediction }\end{array}$ & & $\begin{array}{l}.64 \\
.73 \\
.69 \\
.69\end{array}$ & $\begin{array}{l}.75 \\
.52 \\
.63 \\
.59\end{array}$ & $\begin{array}{l}.48 \\
.53 \\
.50 \\
.52\end{array}$ & $\begin{array}{l}.34 \\
.47 \\
.41 \\
.48\end{array}$ & & $\begin{array}{l}.89 \\
.80 \\
.85 \\
.77\end{array}$ & $\begin{array}{l}.77 \\
.80 \\
.79 \\
.79\end{array}$ & $\begin{array}{l}.89 \\
.79 \\
.84 \\
.80\end{array}$ & $\begin{array}{l}.87 \\
.85 \\
.86 \\
.79\end{array}$ \\
\hline 2 & $\begin{array}{l}\text { Subject } 3 \\
\text { Subject } 4 \\
\text { Average } \\
\text { Prediction }\end{array}$ & & & $\begin{array}{l}.76 \\
.76 \\
.76 \\
.77\end{array}$ & $\begin{array}{l}.70 \\
.77 \\
.73 \\
.69\end{array}$ & $\begin{array}{l}.79 \\
.61 \\
.70 \\
.63\end{array}$ & & & $\begin{array}{l}.79 \\
.73 \\
.76 \\
.79\end{array}$ & $\begin{array}{l}.87 \\
.75 \\
.81 \\
.78\end{array}$ & $\begin{array}{l}.93 \\
.79 \\
.83 \\
.76\end{array}$ \\
\hline 3 & $\begin{array}{l}\text { Subject } 3 \\
\text { Subject } 4 \\
\text { Average } \\
\text { Prediction }\end{array}$ & & & & $\begin{array}{l}.82 \\
.76 \\
.79 \\
.76\end{array}$ & $\begin{array}{l}.73 \\
.57 \\
.65 \\
.69\end{array}$ & & & & $\begin{array}{l}.80 \\
.76 \\
.78 \\
.77\end{array}$ & $\begin{array}{l}.71 \\
.68 \\
.70 \\
.75\end{array}$ \\
\hline
\end{tabular}

*Cell entries are based on 56 observations per subject. 
$(Z=8.4, p<.001, \quad M S E=.015)$, but not for "yes" responses. The effects of response alternative and homogeneity were insignificant.

For the forced-choice task, the effects of display size, target redundancy, and homogeneity were insignificant, but significant interactions were observed between response alternative (" $C$ " vs. " $G$ ") and target redundancy $(Z=2.4, p<.01, \mathrm{MSE}=.015)$, as well as between response alternative and homogeneity $(Z=3.6, p<.001, \mathrm{MSE}=.005)$. Further analysis indicated that the target-redundancy effect was significant for " $C$ " responses (detection accuracy increased from .62 to .80 and .79 as the number of targets increased; $Z=2.8, p<.01, \mathrm{MSE}=.015$ ), but not for " $G$ " responses (mean proportions were $.76, .77$, and .73 ). In addition, " $C$ "' responses were more accurate than " $G$ " responses for the homogeneous condition (.80 compared to $.70 ; Z=2.4$, $\mathrm{p}<.01, \mathrm{MSE}=.004$ ), whereas " $\mathrm{G}$ " responses were more accurate for the heterogeneous condition (.79 compared to $.67 ; \mathrm{Z}=2.7, \mathrm{p}<.01, \mathrm{MSE}=.005)$.

In order to estimate parameters for the three-state model, the observed response probabilities, averaged over subjects, served as input to an iterative gridsearch and hill-climbing program (Reed, 1976). The program determined the set of parameter values which minimize the sum of squared deviations between observed and predicted response probabilities. The representativeness of the solution for the averaged data was assessed by summing the discrepancies between predicted accuracy scores $^{3}$ and the empirical accuracy measures for individual subjects. For the yes-no task, the summed discrepancies were significant between individual subjects' data and the average prediction $\left[\chi^{2}(15)=151, p<.01\right]$, whereas, for the forced-choice task, the discrepancies were insignificant $\left[\chi^{2}(75)=54.7\right]$. Consequently, the yes-no model was fit to the data for each subject individually. The discrepancies between observed and predicted proportions were insignificant for individual subject scores $\left[\chi^{2}(5)=3.85\right.$ and 3.13 for Subjects 1 and 2, respectively]. The predictions of the three-state model are included in Tables 1 and 2 , and the best-fitting parameter estimates, as determined by the grid-search procedure, ${ }^{4}$ are listed in Table 3.

To test whether the pattern of response probabilities predicted by the model corresponded to the effects obtained for empirical proportions, the same statistical analyses that were applied to observed proportions were applied to predicted scores. For the yes-no task, the effect of display size for predicted proportions was significant for both subjects when arrays contained no targets $(Z=2.76, p<.01$, MSE $=.06$, and $Z=5.45, \mathrm{p}<.001$, MSE $=.02$, for Subject 1 and Subject 2, respectively), but, for target
Table 3

Best-Fitting Parameter Estimates for the Three-State Model

\begin{tabular}{|c|c|c|c|c|c|c|c|c|c|}
\hline & $p_{11}$ & $p_{12}$ & $\mathrm{p}_{22}$ & $\mathrm{p}_{21}$ & $p_{31}$ & $p_{32}$ & $\mathrm{p}_{41}$ & $\dot{p}_{42}$ & $\alpha$ \\
\hline & \multicolumn{9}{|c|}{ Yes-No Task* } \\
\hline \multirow[t]{3}{*}{$\begin{array}{l}\text { Subject } 1 \\
\text { Subject } 2\end{array}$} & $\begin{array}{l}.35 \\
.00\end{array}$ & $\begin{array}{l}.00 \\
.00\end{array}$ & $\begin{array}{l}.95 \\
.83\end{array}$ & $\begin{array}{l}.00 \\
.08\end{array}$ & & & & & $\begin{array}{l}.72 \\
.67\end{array}$ \\
\hline & \multicolumn{9}{|c|}{ Forced-Choice Task $\uparrow$} \\
\hline & .53 & .00 & .62 & .12 & .06 & .09 & .00 & .24 & .59 \\
\hline
\end{tabular}

${ }^{*} s_{1}=$ " $C$," $s_{2}=$ either " $G$ " or " $O$."

$t_{1}=" C, " s_{2}=" G, " s_{3}=" O, " s_{4}=$ "Q $Q$ "

arrays, the effect of display size was significant only for Subject $2(Z=2.5, p=.06$, by one-tailed test, MSE $=.02$ ). Since distinct target-array effects were predicted for the two subjects, the observed response probabilities for target arrays were reanalyzed for each subject individually. The effect of display size was significant for Subject $2(\mathrm{Z}=1.7, \mathrm{p}=.05$, by onetailed test, MSE = .03), but not for Subject 1. For the forced-choice task, the effects observed for empirical proportions were also evident for predicted proportions: significant interactions were obtained between response type and target redundancy $(\mathrm{Z}=$ $11.0, \mathrm{p}<.001, \mathrm{MSE}=.01$ ), as well as between response type and homogeneity $(Z=3.5, p<.001$, MSE $=.005$ ).

\section{DISCUSSION}

\section{Yes-No}

For the yes-no task, the display-size effect observed here parallels the results of the previous study (Eriksen \& Spencer, 1969), in that average detection accuracy decreases as display size increases. However, in contrast to previous results, the target-array effect occurred for only one subject (Subject 2) in the present study. The results for Subject 2 agree with predictions based on the confusion-error hypothesis as proposed by Eriksen and Spencer, since, on the basis of their hypothesis, a target-array effect is expected whenever the display-size effect occurs. In contrast, the results for Subject 1 conflict with expectations based on this hypothesis, since a display-size effect was observed for Subject 1 , but the target-array effect was not. The individual differences observed here, then, suggest that the confusion-error hypothesis proposed by Eriksen and Spencer is overly restrictive; confusions between distractors and targets appear to be sufficient but not necessary for the display-size effect to occur.

Nevertheless, the confusion-error hypothesis need not be rejected altogether; the results reported here may be reconciled with Eriksen and Spencer's hypothesis by allowing the possibility that the identity of individual characters is indeterminate. That is, 
rather than assuming only two outcomes of perceptual processing-correct identifications and misidentifications-one can assume that characters are identified as neither target nor distractor. If detection accuracy is negatively correlated with degree of indeterminacy, as is the case in the three-state model, for example, a display-size effect may occur even when the target-array effect does not, since the effects of indeterminacy may differ for the two responses.

The effects of indeterminacy, as defined in the three-state model, may be clarified by examining the fit of the model to the yes-no results. For Subject 1, the probability of misidentifying a distractor as a target $\left(p_{21}\right)$ was estimated to be zero and the probability of correctly identifying a distractor $\left(p_{22}\right)$ was estimated to be less than one. Hence, the probability of indeterminacy $\left(p_{20}=1-p_{12}-p_{22}\right)$ was estimated to be nonzero. Consequently, the probability of a determinate "no" response which is correct, $\mathrm{P}\left(\mathrm{D}_{2} / \mathrm{S}_{2}\right)$, is predicted to decrease as display size increases (Equation 4), the probability of a determinate "yes" response, $P\left(D_{1} / S_{2}\right)$, is predicted to be zero (Equation 3), and the predicted probability of an indeterminate detection for nontarget arrays, $P\left(D_{0} / S_{2}\right)$, increases as display size increases. Thus, for Subject 1 , a display-size effect is expected for nontarget arrays. For target arrays, however, since both $p_{12}$ and $p_{21}$ are estimated to be zero, the value of $P\left(D_{2} / S_{1}\right)$ is predicted to be zero, and $P\left(D_{1} / S_{2}\right)$ is predicted to be constant as display size varies. Hence, $P\left(D_{0} / S_{t}\right)$ is predicted to be constant as display size varies, i.e., no target-array effect is predicted for Subject 1.

In contrast, the parameter estimates for Subject 2 indicate that the probability of confusing a distractor as a target is nonzero $\left(p_{21}\right.$ estimated to be .08$)$, and because of this, $P\left(D_{1} / S_{1}\right)$ is predicted to increase as display size increases. In addition, since $\mathrm{p}_{12}$ is estimated to be zero, $P\left(D_{2} / S_{1}\right)$ is zero and a target-array effect is predicted for Subject 2. Similarly, confusions between distractors and the target contribute to the display-size effect for nontarget arrays, since $P\left(D_{2} / S_{2}\right)$ is predicted to decrease as a function of $\mathbf{N}$. However, stimulus uncertainty contributes to the display-size effect as well, since $p_{20}=1-p_{21}-p_{22}=.09$ is estimated to be nonzero, and, as a result, $P\left(D_{0} / S_{2}\right)$ increases as display size increases. To summarize, both confusion errors and stimulus uncertainty appear to contribute to the display-size effect for Subject 2, whereas, for Subject 1, the display-size effect appears to be due solely to increased stimulus uncertainty. In terms of the three-state model, then, either stimulus uncertainty or misidentifications may be responsible for the display-size effect. Moreover, the effects of misidentifications may be distinguished from the effects of stimulus uncertainty by means of the presence or absence of the target-array effect.
Alternatively, the results observed here may be interpreted in terms of the confusability model proposed by Kinchla (1974). The confusability model is similar to the three-state model in that independent processing is assumed. In contrast to the three-state model, however, Kinchla characterizes the outcomes of perceptual processing in terms of a continuous quantity, representing the degree of confusability between each character and the target. In the model, the degree of confusability (the confusability coding) is summed over all characters in the array to form a "cumulative coding." A subject's response is determined by comparing the cumulative coding to a response criterion, as in signal-detection theory. A display-size effect is predicted by the confusability model, since the variance $\left(\sigma^{2}\right)$ associated with cumulative coding is assumed to increase linearly as display size increases.

To fit the confusability model to Eriksen and Spencer's results, Kinchla (1974) estimated $\sigma^{2}$ for each display size, and then evaluated the linearity of the function relating the variance estimates to display size. In accordance with predictions, $\sigma^{2}$ increased linearly as $\mathbf{N}$ increased. Similarly, a satisfactory fit $^{5}$ was obtained by applying the confusability model to the observations reported here $\left[\chi^{2}(3)=3.0\right.$ for both subjects]. Thus, with respect to changes in coding variance, the predictions of the confusability model are consistent with results from the current study as well as with those reported by Eriksen and Spencer.

The predictions of the confusability model are consistent as well with the qualitative trends observed here, provided that an assumption is introduced regarding changes in the mean response criterion, $\beta$, as a function of display size. The constraints on $\beta$ required by existing results can be seen by noticing that the probability of a correct detection for target arrays (hit rate) is always observed to be greater than .5 . Consequently, the confusability model requires that $\beta<\mathrm{t}$, where $\mathrm{t}$ is the mean confusability coding for target arrays. If $\sigma^{2}$ increases, then, $\beta$ must decrease as display size increases in order for the target-array effect to occur. Thus, the target-array effect reported by Eriksen and Spencer, and observed for Subject 2 in the present study, is consistent with the confusability model, provided that the increase in hit rate resulting from decreased $\beta$ exceeds the decrease in hit rate resulting from increased $\sigma^{2}$. Similarly, the results for Subject 1 in the present study are consistent with the confusability model, provided that the decrease in $\beta$ compensates exactly for the increase in $\sigma^{2}$, so that the hit rate remains constant as display size varies.

In summary then, a satisfactory fit was found between predictions of both the confusability and three-state models to the yes-no results observed here. The two models differ in terms of their assump- 
tions regarding the decision process, and, because of this, some features of the results are subject to alternative interpretations. Nevertheless, the independence assumption is required by both models and, therefore, independent processing, in combination with either decision model, appears to offer a satisfactory account of the yes-no results.

\section{Forced-Choice}

The effects of target redundancy and homogeneity observed for the forced-choice task differ from previous studies in that the interactions observed here between target type and other display variables were not reported previously. In addition, the current results differ from previous reports in that the display-size effect in the current study was insignificant.

The interactions between target type and display characteristics (target redundancy and homogeneity) are understandable in terms of inequalities in classification probabilities among stimuli. That is, since the interactions were evident for predicted proportions as well as observed proportions, the inequalities as measured by the parameter estimates for the threestate model appear to be sufficient to account for the observed results. Both the interaction between target redundancy and response alternative and the accuracy advantage for "C" responses in the homogeneous condition are consistent with the asymmetry of the classification probabilities for both subjects (i.e., $\mathrm{p}_{21}>\mathrm{p}_{12}=0$ ) and the response bias in favor of " $C$ " responses $(\alpha=.59)$. Thus, the interaction between target redundancy and response alternative may be attributed to an increase in the probability of detecting at least one " $\mathrm{C}$ " as the number of Gs increases, whereas the probability of detecting at least one " $G$ " does not increase with the number of Cs. Similarly, the accuracy advantage observed for " $G$ " responses in the heterogeneous condition, and for " $C$ " responses in the homogeneous condition, is consistent with the inequality in parameter estimates for $p_{42}$ and $p_{41}$.

The absence of a display-size effect for the forcedchoice results was unanticipated, however. A displaysize effect was expected in the present study, both because of the prevalence of the effect in the case when targets and distractors are similar (Estes, 1972; Estes \& Taylor, 1964, 1966; Gardner, 1973), and because of the attempt to maximize target-distractor similarity and display size. Yet, the effects of inequalities in similarity among distinct target-distractor pairs was not considered previously. As a result, it is possible that the effect of display size would be insignificant when averaged over target-distractor pairs but significant for individual target-distractor combinations. For example, consider the case when $S_{2}$ is more similar than $S_{1}$ to the distractor, $S_{3}$. In this case, the magnitude of the display-size effect is expected to be greater for displays which contain $S_{1}$ and $S_{3}$ than for displays containing $S_{2}$ and $S_{3}$, since, as display size increases, the occurrence of misidentifications will act to increase $\mathbf{P}\left(\mathbf{R}_{2}\right)$ relative to $\mathbf{P}\left(\mathbf{R}_{1}\right)$. In fact, as the similarity between $S_{2}$ and $S_{3}$ increases, the magnitude of the display-size effect for $R_{2}$ decreases, and, in the limit, $P\left(R_{2}\right)$ may actually increase as display size increases. In the case of unequal similarity between the two target-distractor combinations, then, the effects of display size are expected to differ for the two responses: a displaysize effect is expected for the dissimilar targetdistractor pair (in contrast to the expectations for averaged data); and the effect may or may not occur for the similar pair.

To test this possibility, the effects of display size were examined for each of the four possible target and distractor combinations included in the present study. The display-size effect was significant for " $C$ " responses when " $O$ " and " $Q$ " served as distractors ( $\mathrm{Z}$ for linear trend $=1.8, \mathrm{p}<.05, \mathrm{MSE}=.03$ ), and for " $G$ " responses when " $O$ " served as the distractor ( $Z$ for quadratic trend $=1.7, p<.05$, MSE $=.02$ ). The effects of display size were insignificant for the two remaining categories.

The display-size effect observed for " $C$ " responses in the heterogeneous condition is consistent with expectations based on the effects of unequal similarity among target-distractor pairs, since the pair $(G, Q)$ was estimated to be more similar than the pair $(C, Q)$ and the display-size effect was significant for predicted as well as observed proportions $(Z=1.7, p<$ .05 , MSE $=.03$ ). In contrast, the display-size effect observed for " $G$ " responses is inconsistent with expectations based on the parameter estimates and corresponding predictions of the three-state model; the similarity between " $\mathrm{C}$ " and " $\mathrm{O}$ " was estimated to be approximately equal to that for " $G$ " and " $O$," and no display-size effect is evident for predicted proportions.

With one exception (the observed display-size effect for " $G$ " responses), then, the results observed for the forced-choice task are consistent with the hypothesis that individual characters are processed independently, and that changes in detection accuracy in relation to display characteristics (i.e., display size, target redundancy, homogeneity) may be accurately summarized in terms of the three-state model.

Some indication of the uniqueness of these results may be obtained by comparing the three-state model to alternative models of the forced-choice task (Estes, 1976; Gardner, 1973; Shiffrin \& Geisler, 1973). The model proposed by Gardner is most similar to the three-state model in that (1) the outcomes of perceptual processing are represented in terms of three discrete endstates, and (2) the decision process is "all-or-none," i.e., whenever a state of indeterminacy 
exists, response selection is based solely on response bias. Consequently, a generalization of Gardner's model to the case when distinct effects are observed for the two response alternatives is likely to be indistinguishable from the three-state model. For this reason, Gardner's model was not applied to the present results.

The model proposed by Shiffrin and Geisler requires specific assumptions regarding the effective features. Specifically, the model has only been applied to the case when stimulus characters are defined by discrete points (as letters are displayed on a CRT). Since the characters displayed here were defined by continuous contours, then, the Shiffrin and Geisler model would appear to be inapplicable.

The model proposed by Estes (1976), however, is applicable to a partial set of the present results. Estes' (1976) model was not intended to apply to the case when distinct effects are observed for each of the response alternatives. But the model can be compared to the three-state model in order to test encoding, and response-selection assumptions, since, in the Estes (1976) model, (1) perceptual processing is represented in terms of a feature-activation process, rather than in terms of discrete states; and (2) a "proportional," rather than an "all-or-none," response rule is assumed, i.e., response selection in the case of uncertainty is based on the proportion of features consistent with each response.

To compare the two models, the response proportions observed here were averaged over homogeneity and target type. The grid-search procedure discussed previously was then used to determine the parameter values for each model which minimized the sum of squared deviations between observed and predicted response proportions. A satisfactory fit was obtained for both the feature-activation model $\left[\chi^{2}(10)=6.9\right]$ and a three-parameter version of the three-state model $^{3}\left[\chi^{2}(9)=3.7\right]$. In terms of goodness-of-fit for the averaged results, then, both the feature-activation and three-state models are consistent with the results for the forced-choice task. Furthermore, since the fit of the three-state model was satisfactory for both the complete set of results and the averaged results, the independence assumption, in combination with a model of encoding and response selection, appears to be sufficient to account for the forced-choice results reported here.

In conclusion, for both yes-no and forced-choice tasks, satisfactory agreement was obtained between observed values of detection accuracy and predictions based on the three-state model. In addition, the effects obtained for observed proportions accorded well with effects predicted by the model, and accuracy differences between subjects were consistent with the parameter estimates for each subject. (Goodnessof-fit was also satisfactory for alternative detection models incorporating the independence assumption.)
The independence assumption, in combination with a three-state model of the detection process, then, appears sufficient to account for the effects of display characteristics on detection accuracy observed in the current study. Hence, in order to measure perceptual span, experimental conditions must be found which place a greater demand on processing resources than those investigated here.

\section{REFERENCES}

Atrinson, R. C., \& Kinchla, R. A. A learning model for forcedchoice detection experiments. British Journal of Mathematical and Statistical Psychology, 1965, 18, 183-206.

BoumA, H. Interaction effects in parafoveal letter recognition. Nature, 1970, 226, 177-178.

Cattell, J. McK. Über die Trägheit der Netzhaut und des Sehcentrums. Philosophische Studien, 1883, 3, 94-127.

Collins, J. F., \& Eriksen, C. W. The perception of multiple, simultaneously presented forms as a function of foveal spacing. Perception \& Psychophysics, 1967, 2, 369-373.

Cox, D. R. The analysis of binary data. London: Methuen, 1970.

ERIKSEN, C. W., \& SPENCER, T. Rate of information processing in visual perception: Some results and methodological considerations. Joumal of Experimental Psychology Monographs, 1969 , 79(2, Pt. 2).

Estes, W. K. Interactions of signal and background variables in visual processing. Perception \& Psychophysics, 1972, 12, 278-286.

EsTES, W. K. Perceptual processing in letter recognition and reading. In E. C. Carterette \& M. P. Friedman (Eds.), Handbook of perception, (Vol. IX). New York: Academic Press, 1976.

Estes, W. K., \& TAYLOR, H. A. A detection method and probabilistic model for assessing information processing from brief visual displays. Proceedings of the National A cademy of Sciences, 1964 , 52, 446-454.

Estes, W. K., \& TAylor, H. A. Visual detection in relation to display size and redundancy of critical elements. Perception \& Psychophysics, 1966, 1, 9-16.

Fisher, D. F., Monty, R. A., \& Glucksberg, S. Visual confusion matrices: Fact or artifact? Journal of Psychology, 1969, 71, 111-125.

GARDNER, G. T. Evidence for independent parallel channels in tachistoscopic perception. Cognitive Psychology, 1973, 4, 130-155.

Halton, J. H. An interpretation of negative probabilities. Proceedings of the Cambridge Philosophical Society, 1966, 62, 83-86.

Holmgren, J. E. Visual search with imperfect recognition. Perception \& Psychophysics, 1968, 4, 247-252.

JAMES. W. Principles of psychology (Vol. I). New York: Holt, 1890.

KinCHLA, R. A. Detecting target elements in multielement arrays: A confusability model. Perception \& Psychophysics, 1974, 15, 149-158.

Norman, D. A., \& Bobrow, D. G. On data-limited and resourcelimited processes. Cognitive Psychology, 1975, 7, 44-64.

REED, A. V. Modpac: Modular program package for curve fitting and plotting. Decus Program Library, 1976, 15-92.

RUMELFART, D. E. A multicomponent theory of the perception of briefly exposed visual displays. Joumal of Mathematical Psychology, 1970, 7, 19i-218.

SHIFFRIN, R. M., \& GARONER, G. Visual processing capacity and attentional control. Journal of Experimental Psychology, 1972, 93. $72-82$.

SHIFFRIN, R. M., \& GeISLER, W. S. Visual recognition in a theory of information processing. In R. L. Solso (Ed.), Contemporary issues in cognitive psychology: The Loyola Symposium. New York: Wiley, 1973. Pp. 53-101.

SPERLING, G. The information available in brief visual presentations. Psychological Monographs, 1960, 74(Whole No. 498). 
TownsEnd, J. T. Theoretical analysis of an alphabetic confusion matrix. Perception \& Psychophysics, 1971, 9, 40-50.

\section{NOTES}

1. An alternative definition of state $D_{2}$ is that $P\left(D_{2}\right)$ equals the proportion of channels which register as State 2, provided that no channels register as State 1 (D. Noreen, personal communication). The "proportional" definition of $\mathrm{D}_{2}$ yielded goodness-of-fit estimates which were similar to those obtained using Equation 4.

2. Test statistics are based on a logistic transformation of response probability, defined as: $h=\log [(r+.5) /(n-r+.5)]$, where $r$ equals the frequency of correct responses and $n$ equals the number of observations. The statistic $h$ is normally distributed (Cox, 1970) with variance, $V$, equal to $[(n+1)(n+2)] /[n(r+1)$ $(n-r+1)]$

3. The statistic $\chi^{2}(\mathbf{N}-p)$ was defined as

$$
\sum_{j=1}^{2} \sum_{i=1}^{N} \frac{\left(h_{i j}-\hat{h}_{i j}\right)^{2}}{\hat{V}_{i j}} \text {. }
$$

where $p$ equals the number of parameters estimated, $h_{i j}$ is the logistic transform of the observed proportion, and $\hat{h}_{\mathrm{ij}}$ is the logistic transform of the predicted proportion, $\mathrm{N}$ is the number of data points, the subscript $i$ indexes the conditions, and $j$ indexes the two subjects.

4. Parameter estimates were constrained to be nonnegative. When this constraint was removed, the estimates of $p_{12}$ for the yes-no task and $\mathrm{p}_{12}$ and $\mathrm{p}_{41}$ for the forced-choice task assumed negative values. Since the sum of squared deviations differed by less than .01 for the two cases, and the occurrence of negative probabilities need not imply rejection of the model (Halton, 1966), only the nonnegative parameter estimates are reported.
5. The goodness-of-fit statistic used to test the confusability model was defined as:

$$
\chi_{(3)}^{2}=\sum_{i=1}^{s} \frac{\left(\frac{1}{D^{2}}-\frac{1}{D^{2}}\right)^{2}}{S_{y \cdot x}^{2}},
$$

where $1 / \mathrm{D}^{2}$ and $1 / \hat{\mathrm{D}}^{2}$ are the observed and predicted values of coding variance, and $S_{y \cdot x}^{2}$ is the sample variance of estimate associated with the prediction.

Appendix

Mean Response Times for the Yes-No and Forced-Choice Tasks

Display Size

\begin{tabular}{lcccccccccc} 
& \multicolumn{10}{c}{ Display Size } \\
\cline { 2 - 9 } & 1 & 2 & 4 & 6 & 8 & 1 & 2 & 4 & 6 & 8 \\
\hline \multicolumn{1}{c}{ Yes-No Task } \\
Correct & 529 & 521 & 526 & 524 & 550 & 566 & 582 & 598 & 600 & 622 \\
Error & 582 & 588 & 590 & 580 & 590 & 578 & 580 & 589 & 597 & 611
\end{tabular}

Forced-Choice Task

Target

Frequency

Correct Responses

Errors

1

533535539528542

514519529516

$595 \quad 575566554582$

$512 \quad 522 \quad 518$

$\begin{array}{llll}533 & 525 & 535 & 560\end{array}$

3

(Received for publication March 16, 1978; revision accepted September 20, 1978.) 